Methods: This is a cross-sectional, observational, and comparative study. A total of seventy-five PsA patients aged 40-75 years old, who fulfilled the 2006 CASPAR criteria and seventy-five matched controls by age ( \pm 5 years), gender and comorbidities were recruited for this study. Patients with history of a previous CV event and pregnant women were excluded from this study. A high-resolution $B$ mode carotid US was performed in all study subjects by a certified radiologist. Subclinical atherosclerosis was defined as the presence of a carotid plaque (CP) or an increased carotid intima media thickness (cIMT). The presence of CP was defined as a cIMT $\geq 1.2 \mathrm{~mm}$ or a focal narrowing $\geq 0.5 \mathrm{~mm}$ in the surrounding lumen. An increased cIMT was considered as a value $\geq 0.8 \mathrm{~mm}$. Distribution was evaluated with the Kolmogorov-Smirnov test. Comparisons were done with $\chi 2$ test for qualitative variables and Student's t test and Mann-Whitney's $U$ test for quantitative variables. A $p$ value $<0.05$ was considered statistically significant. Results: There were no differences when comparing the demographic characteristics between both groups (Table 1). When comparing the carotid US findings, a statistically significant difference was found in the prevalence of $\mathrm{CP}$, which was higher in the PsA group (44.0\% vs $26.7 \%, p=0.026$ ), in the presence of unilateral CP $(25.3 \%$ vs $10.7 \%, p=0.019)$ and in the presence of subclinical atherosclerosis (52.0\% vs $34.7 \%, p=0.032$ ) (Figure 1 ).

Conclusion: The prevalence of subclinical atherosclerosis was higher in PsA patients than controls, and this could be attributed to an increase in the inflammatory burden of these patients. The carotid US should be considered as part of the CV evaluation in all PsA patients, identifying those who would benefit from an opportune treatment preventing the development of a CV event.

REFERENCES:

[1] Yim KM, Armstrong AW. Updates on cardiovascular comorbidities associated with psoriatic diseases: epidemiology and mechanisms. Rheumatol Int. 2017;37(1):97-105.

Table 1. Demographic and clinical characteristics of psoriatic arthritis patients and controls.

\begin{tabular}{|c|c|c|c|}
\hline & $\begin{array}{l}\text { PsA } \\
(n=75)\end{array}$ & $\begin{array}{l}\text { Controls } \\
(n=75)\end{array}$ & $p$ \\
\hline Age years, mean $\pm \mathrm{DE}$ & $53.89 \pm 10.59$ & $54.25 \pm 7.08$ & NS \\
\hline Female gender, $\mathrm{n}(\%)$ & $43(57.3)$ & $43(57.3)$ & NS \\
\hline T2DM, n (\%) & $16(21.3)$ & $15(20.0)$ & NS \\
\hline HTN, n (\%) & $28(37.3)$ & $21(28.0)$ & NS \\
\hline Dyslipidemia, n (\%) & $33(44.0)$ & $28(37.3)$ & NS \\
\hline Obesity, n (\%) & $31(41.3)$ & $32(42.7)$ & NS \\
\hline Active smoking, n (\%) & $14(18.7)$ & $18(24.0)$ & NS \\
\hline BMI kg/m², median (p25-p75) & $29.32(26.23-32.03)$ & $28.9(25.4-33.5)$ & NS \\
\hline Disease duration years, median (p25-p75) & $5.0(3.0-10.0)$ & - & - \\
\hline DAPSA, median (p25-p75) & $12.6(5.3-22.9)$ & - & - \\
\hline Glucocorticoids, $n(\%)$ & $10(13.3)$ & - & - \\
\hline MTX, n (\%) & $51(68.0)$ & - & - \\
\hline bDMARD, n (\%) & $28(37.3)$ & - & - \\
\hline
\end{tabular}

PsA, psoriatic arthritis; NS, not significant; T2DM, type 2 diabetes mellitus; HTN, hypertension; BMI, body mass index; DAPSA, disease activity for psoriatic arthritis; MTX, methotrexate; bDMARD, biological disease modifying antirheumatic drug

Figure 1. Carotid ultrasound findings in psoriatic arthritis patients and controls.

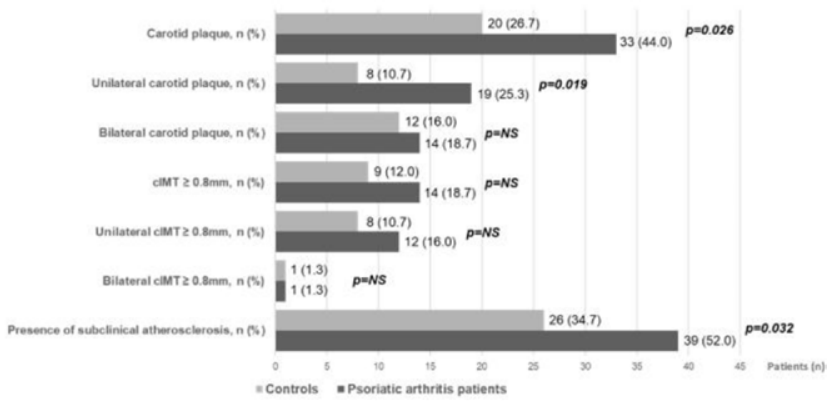

Disclosure of Interests: None declared

DOI: 10.1136/annrheumdis-2021-eular.3188

\section{POS1403 RADIOSYNOVECTOMY IN A CLINICAL SETTING: AN UNDEREXPLOITED TOOL AT OUR DISPOSAL}

L. R. Caballero Motta ${ }^{1}$, A. M. Anzola Alfaro ${ }^{1}$, J. Molina Collada ${ }^{1}$, I. Janta ${ }^{1}$, Y. K. Henao ${ }^{2}$, R. Perez Pascual ${ }^{2}$, F. J. López-Longo ${ }^{1}$, J. M. Alvaro Gracia ${ }^{1}$, J. C. Nieto González ${ }^{1}{ }^{1}$ Gregorio Marañón Hospital, Rheumatology, Madrid, Spain;

${ }^{2}$ Gregorio Marañón Hospital, Nuclear Medicin, Madrid, Spain
Background: Radiosynovectomy (RS) is a useful for treating inflammatory arthritis that fail conventional treatments. The main isotope used is Yttrium-90 on large joints as knees, whereas Erbium-169 and Renium-186 are more common in small and medium sized joints respectively.

$\mathrm{RS}$ is a safe procedure since the isotopes cannot escape the synovial capsule or be absorbed into circulation. It is, however, lethal against cells within the inflamed joint. The most common rheumatic disease treated with RS is rheumatoid arthritis (RA), followed by axial spondyloarthritis $(\mathrm{SpA})$ and idiopathic juvenile arthritis (JIA). It has also been used on persistent synovitis after joint replacements, pigmented villonodular synovitis (PVNS) and undifferentiated arthritis.

Objectives: To describe the experience in RS of a tertiary rheumatology center and compare patients with and without clinical response to treatment in the following 12 months. Methods: Observational retrospective study between May 31st 2013 and October 31st 2019. We collected demographic variables, data about the disease of the patient, the joints affected, isotope utilized, presence of Baker's cyst, systemic treatment received, need of additional infiltrations (before and after), complications and any changes in medication up to a year after the procedure.

All the RS were performed ambulatory and the radioisotope infiltration was guided by ultrasound, with $40 \mathrm{mg}$ of triamcinolone infiltrated after.

SPSS v23 was used for statistical analysis; with Chi2 for qualitative variables and Student's T distribution for quantitative variables.

Results: We evaluated 67 joints in 49 patients in total. All of them were refractory to conventional treatment. 44 patients $(65.7 \%)$ were women, median of 53.4 years of age (IQ 43.4-67.1)

The median disease duration was 12.5 years and RS seemed to fare better the longer the patient had the disease (median of 13.5 years vs 6.5 years $p<0.001$ ). The joints infiltrated where $46(68.6 \%)$ knees, $14(20.9 \%)$ wrists and $7(15.2 \%)$ elbows. Out of the knees, 16 (34.8\%) belonged to RA patients with effective response in $14(87,5 \%) .100 \%$ of elbows had an effective response, of them 6 $(85.7 \%)$ had RA. However, even when $9(64.2 \%)$ wrists also had RA as diagnosis, only $3(21.4 \%)$ were effective.

Of the PVNS, 6 out of 8 (75\%) had no clinical response, as shown in Table 1.

Table 1. RS response compared to clinical diagnosis.

\begin{tabular}{lllll}
\hline & TOTAL & EFFECTIVE INEFFECTIVE p \\
\hline & 67 & $46(68.6 \%)$ & $21(31.3 \%)$ & \\
& $(100 \%)$ & & & \\
Inflammatory Arthritides & $52(77.6)$ & $\mathbf{3 9}(\mathbf{7 5 \% )}$ & $13(25 \%)$ & $<0.0001$ \\
& & & & \\
(RA + PsA + SpA + SJIA), (\%) & & & & \\
RA (\%) & $30(44.7)$ & $\mathbf{2 2}(\mathbf{7 3 . 3})$ & $8(26.6)$ & $<0.001$ \\
RA positive ACPA/FR & $21(70)$ & $\mathbf{1 5}(\mathbf{7 1 . 4 )}$ & $6(28.6)$ & $<0.0001$ \\
Psoriasic arthritis (PsA) (\%) & $6(9)$ & $4(66.6)$ & $2(33.3)$ & 0.42 \\
SpA (\%) & $10(14.9)$ & $8(80)$ & $2(20)$ & 0.45 \\
sJIA (\%) & $6(9)$ & $5(83.3)$ & $1(16.6)$ & 0.55 \\
PVNS (\%) & $8(11.9)$ & $2(25)$ & $6(75)$ & $<0.001$ \\
Inespecific monoarthritis (\%) & $3(4.4)$ & $3(100)$ & $0(0)$ & 0.23 \\
OA + Calcium Pyrophosphate Deposition & $4(5.9)$ & $2(50)$ & $2(50)$ & 0.33 \\
(CPPD) (\%) & & & & \\
\hline
\end{tabular}

Intra articular corticosteroids were needed before RS, with no differences in effective and ineffective joints; however after RS it was significantly lower in effective joints in the first six months ( $0 \%$ vs $43 \% p<0.0001)$ and remained so in the following 6 months ( $0 \%$ vs $19 \% p<0.0001)$ Only $13(28 \%)$ patients with effective RS needed to change systemic treatment compared to 10 $(43 \%)$ of those ineffective $(p<0.0001)$. None of the patients with RS had any complication after the procedure during follow up.

Conclusion: Our study showed that knees were the main joint infiltrated and they had an overall good response to treatment, especially if the diagnosis was RA. Patients with effective procedures needed leest treatment changes and significantly less corticosteroids infiltrations.

In our study, RS in PVNS was significantly less effective than in inflammatory arthritis ( $25 \%$ vs $75 \% \mathrm{p}<0.0001$ ) and RA seemed to have the best response overall.

\section{REFERENCES:}

[1] Liepe K. Efficacy of radiosynovectomy in rheumatoid arthritis. Rheumatol Int. 2012 Oct; 32(10):3219-24.

[2] Ćwikła JB, Żbikowski P, Kwiatkowska B, Buscombe JR, Sudoł-Szopińska I. Radiosynovectomy in rheumatic diseases. J Ultrason. 2014 Sep; 14(58):241-51.

Disclosure of Interests: None declared

DOI: 10.1136/annrheumdis-2021-eular.3250

\section{POS1404 ECHOCARDIOGRAPHIC FINDINGS IN SYSTEMIC LUPUS ERYTHEMATOSUS PATIENTS AND ITS RELATION TO ANTIBODIES TITERS}

A. B. Rodriguez-Romero ${ }^{1}$, J. R. Azpiri-López ${ }^{2}$, I. J. Colunga-Pedraza ${ }^{1}$, D. Á. Galarza-Delgado ${ }^{1}$, S. Lugo-Perez ${ }^{2}$, N. Guajardo-Jauregui ${ }^{1}$, A. Cárdenas ${ }^{1}$, H. Azpiri-Diaz ${ }^{2}$, O. A. Cepeda-Ayala ${ }^{2} .{ }^{1}$ Hospital Universitario "Dr. José Eleuterio 
González", Universidad Autónoma de Nuevo León, Rheumatology Service, Monterrey, Mexico; ${ }^{2}$ Hospital Universitario "Dr. José Eleuterio González", Universidad Autónoma de Nuevo León, Cardiology Service, Monterrey, Mexico

Background: Systemic lupus erythematosus (SLE) is a chronic and autoimmune disease characterized by systemic involvement. Patients with SLE have accelerated atherosclerosis, resulting in an up to nine-fold increased risk of cardiovascular disease, compared to the general population (1), being the leading cause of death for these patients. Speckle tracking echocardiography (STE) is an accurate technique to estimate myocardial function and deformation.

Objectives: This study aims to determine the association between echocardiographic findings and the presence of antibodies in SLE patients.

Methods: This was a cross-sectional and observational study. A total of forty-three patients $\geq 18$ years with a diagnosis of SLE according to EULAR/ACR 2019 criteria were included for this study. Those with a history of cardiovascular disease (myocardial infarction, cerebrovascular accident, or peripheral arterial disease) and pregnancy were excluded. Transthoracic echocardiogram was performed and reviewed by 2 board-certified cardiologists, in all study subjects. Blood samples obtained from all patients were analyzed for the following: anti-nuclear antibodies (ANA), anti, SSA/Ro, SSB/La antibodies, anti-cardiolipin antibodies (IgA, $\operatorname{lgM}, \lg \mathrm{G})$, and complement levels. Distribution was evaluated with the Kolmogorov-Smirnov test. Correlations between numerical variables were done using Spearman's rho, considering two-tailed $p$-values $<0.05$ as statistically significant. Results: The 39 female patients $(90.7 \%)$ and 4 male patients $(9.3 \%)$ had a mean age of $35.5 \pm 12.0$ years and a median disease duration of 72 months (14-132). At the time of inclusion, $90.7 \%$ of the patients were being treated with glucocorticoids and antimalarials. Concerning traditional cardiovascular risk factors; $20.9 \%$ of the patients had hypertension, $7.0 \%$ had dyslipidemia, $2.3 \%$ had diabetes mellitus and $18.6 \%$ were active smokers. Correlations between echocardiographic findings and antibodies are shown in Table 1. We found a moderate positive correlation between global circumferential strain and $\lg A$ anticardiolipin antibody ( $r=0.507, p=0.002)$, a low positive correlation in left ventricular ejection fraction with anti-Ro $(r=0.397, p=0.012)$ and anti-La $(r=0.397, p=0.012)$ and a low positive correlation between TAPSE and C3 levels $(r=0.396, p=0.013)$.

Conclusion: There is an association between anticardiolipin antibody titers, anti-Ro, and anti-La with echocardiographic alterations. All SLE patients especially those who had positive antibodies should be screened for the presence of structural cardiac abnormalities. STE can be helpful as a noninvasive diagnostic tool, that could result in earlier treatment and prognosis.

REFERENCES:

[1] Hesselvig JH, Ahlehoff O, Dreyer L, et al. Cutaneous lupus erythematosus and systemic lupus erythematosus are associated with clinically significant cardiovascular risk: a Danish nationwide cohort study. Lupus 2017;26(1):4853. doi: 10.1177/0961203316651739

Table 1. Spearman rho correlations between antibody titers and echocardiographic findings.

\begin{tabular}{|c|c|c|c|}
\hline Variables & $\begin{array}{l}\text { GLS, } \\
\text { mean } \pm \text { SD } \\
-19.11 \pm 3.33\end{array}$ & $\begin{array}{l}\text { LVEF, } \\
\text { mean } \pm \text { SD } \\
57.43 \pm 7.17\end{array}$ & $\begin{array}{l}\text { TAPSE, } \\
\text { mean } \pm \text { SD } \\
22.23 \pm 3.24\end{array}$ \\
\hline ANA, median (p25-p75) & NS & NS & NS \\
\hline $\begin{array}{l}640 \text { (160-2550) } \\
\text { IgA Anti-Cardiolipin, median (p25-p75) }\end{array}$ & $0.507^{\star *}$ & NS & NS \\
\hline $2(2-3)$ & & & \\
\hline $\begin{array}{l}\text { IgM Anti-Cardiolipin, median (p25-p75) } \\
2(2-4)\end{array}$ & NS & NS & NS \\
\hline $\begin{array}{l}\text { IgG Anti-Cardiolipin, median (p25-p75) } \\
4(3-6)\end{array}$ & NS & NS & NS \\
\hline $\begin{array}{l}\text { Anti-Ro, median (p25-p75) } \\
17(2-80)\end{array}$ & NS & $0.326^{*}$ & NS \\
\hline $\begin{array}{l}\text { Anti-La, median (p25-p75) } \\
3(2-5.5)\end{array}$ & NS & $0.397^{*}$ & NS \\
\hline $\begin{array}{l}\text { C3, mean } \pm \text { SD } \\
91.41 \pm 37.38\end{array}$ & NS & NS & $0.396^{*}$ \\
\hline
\end{tabular}

${ }^{* *}$ Correlation is significant at the 0.01 level (2-tailed). ${ }^{*}$ Correlation is significant at the 0.05 level. NS, not significant; GLS, global circumferential strain; LVEF, left ventricular ejection fraction; TAPSE, tricuspid annular plane systolic excursion.

Disclosure of Interests: None declared

DOI: 10.1136/annrheumdis-2021-eular.3399

\begin{tabular}{|l|l}
\hline POS1405 & OPTICAL SPECTRAL TRANSMISSION (HANDSCAN) IN \\
& PATIENTS WITH PSORIATIC ARTHRITIS: FIRST DATA ON \\
& DIAGNOSTIC VALUE AND ASSOCIATIONS WITH CLINICAL \\
& AND ULTRASOUND DISEASE ACTIVITY MARKERS
\end{tabular}

K. Triantafyllias ${ }^{1}$, S. Liverakos ${ }^{2}$, C. Noack ${ }^{3}$, A. Schwarting ${ }^{1,2} .{ }^{1}$ ACURA Clinics,

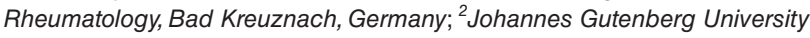
Medical Center, Internal Medicine I, Deparmtnet of Rheumatology and Clinical
Immunology, Mainz, Germany; ${ }^{3}$ University of Mannheim, Graduate School of Economics and Social Sciences, Mannheim, Germany

Background: Valid assessment of disease activity leads to improvement of long-term outcomes in patients with inflammatory arthritis (1). Optical spectra transmission (OST) is a modern diagnostic tool able to assess the blood-specific absorption of light transmitted through a tissue, promising quantification of inflammation in the finger and wrist joints of patients with rheumatoid arthritis (RA) (commercial device: HandScan - Demcon/Hemics, The Netherlands) (2). Even though an increasing number of studies have evaluated diagnostic value of this new technology in RA patients $(2,3)$, no data exist regarding psoriatic arthritis (PSA)

Objectives: To examine for the first time the diagnostic value of OST in detecting inflammation in patients with PsA and to evaluate its relationship with disease activity markers and various epidemiological and anthropometric patient characteristics.

Methods: OST-Measurements were performed in a group of PsA patients and a group of healthy controls. The difference between OST in the two groups was statistically examined and relationships of OST with clinical (tender / swollen joint counts, disease activity on a visual analogue scale) and serological disease activity markers were evaluated. Moreover, joint ultrasound (US) examinations were performed in a subgroup of PSA patients and OST associations with a Power Doppler- and a Grey Scale-US score were examined. Finally, relationships of OST with various anthropometric and epidemiologic parameters (BMI, handsize, gender, age) were assessed.

Results: We recruited 49 PsA patients [65.3\% female; mean age 53.3 years $( \pm 11.8 \mathrm{SD})]$ and 114 control subjects $[77.2 \%$ female; mean age 46 years $( \pm$ 12.8 SD)]. OST was statistically significantly higher in the patient group, compared to the control group [14.95 (12.04 - 17.18, IQR) vs. 10.31 (7.84 - 13.79 IQR); $p<0.001]$. OST correlated moderately-strongly with both examined US scores (Power Doppler-score: $r=0.5 ; p=0.026$ and Grey Scale-score: $r=$ $0.52 ; p=0.028)$. Moreover, OST showed a moderate, statistically significant association with $\mathrm{C}$-reactive protein $(\mathrm{CRP})(r=0,298 ; p=0,037)$. Finally, males had significantly higher OST values than females and OST associated moderately-weakly with body mass index (BMI) in the control group (rho $=0.24$ $\mathrm{p}<0.001)$.

Conclusion: This is the first report of a possible diagnostic value of OST in patients with PSA. OST correlated with ultrasound and serological activity markers and may thus prove to be a useful tool of disease activity assessment, next to well established diagnostic modalities, such as the joint US. Correlations of OST with patient characteristics implicate the need to take also anthropometric and epidemiological patient characteristics into account when interprenting OST results in order to avoid confounding.

\section{REFERENCES:}

[1] Katchamart W, et al. Systematic monitoring of disease activity using an outcome measure improves outcomes in rheumatoid arthritis. J Rheumatol 2010;37:1411-1415.

[2] Triantafyllias, et al. Diagnostic value of optical spectral transmission in rheumatoid arthritis: associations with clinical characteristics and comparison with joint ultrasonography. J Rheumatol 2020 1;47(9):1314-1322.

[3] Onna M Van, et al. Assessment of disease activity in patients with rheumatoid arthritis using optical spectral transmission measurements, a non-invasive imaging technique. Ann Rheum Dis 2016;75:511-518.

Disclosure of Interests: Konstantinos Triantafyllias Speakers bureau: Pfizer, Novartis, Janssen, Chugai, Stefanie Liverakos: None declared, Claudia Noack: None declared, Andreas Schwarting: None declared DOI: 10.1136/annrheumdis-2021-eular.3670

\section{POS1406 \\ DEVELOPMENT OF A DIAGNOSTIC ALGORITHM FOR THE DIFFERENTIAL DIAGNOSIS OF INTERSTITIAL LUNG DISEASE: PRELIMINARY DATA FROM A MULTICENTER RETROSPECTIVE CASE-CONTROL STUDY}

B. Maranini ${ }^{1}$, T. Chiodin ${ }^{1}$, C. A. Scirè ${ }^{1}$, M. Govoni ${ }^{1}$, E. Lucioni ${ }^{2}$, S. Chiarello ${ }^{2}$ F. Scabbia ${ }^{2}$, I. Marchi ${ }^{3}$, G. Zanframundo ${ }^{4}$, L. Cavagna ${ }^{4}$, E. Bellis ${ }^{4}$, M. Silva ${ }^{5}$, G. Tringali ${ }^{5}$, A. Carnevale ${ }^{2} .{ }^{1}$ Rheumatology Unit, University of Ferrara and Azienda Ospedaliero-Universitaria S. Anna, Department of Medical Sciences, Ferrara, Italy; ${ }^{2}$ Radiology Unit, Azienda Ospedaliero-Universitaria S. Anna, Department of Radiology, Ferrara, Italy; ${ }^{3}$ Pneumology Unit, University of Ferrara and Azienda Ospedaliero-Universitaria S. Anna, Department of Medical Sciences, Ferrara, Italy; ${ }^{4}$ University and IRCCS Policlinico S. Matteo Foundation of Pavia, Department of Rheumatology, Pavia, Italy; ${ }^{5}$ Department of Medicine and Surgery (DiMeC), University of Parma, Scienze Radiologiche, Parma, Italy

Background: Interstitial lung diseases (ILDs) represent a heterogeneous group of disorders with different treatment and prognosis. ILD may be the presenting or 